
\title{
3 Research Square \\ Persistently Gadolinium Enhancing Lesion is Predictor of Poor Prognosis in NMOSD Attack: A Clinical Trial
}

\section{Yun Xu}

Beijing Tiantan Hospital

\section{Xindi Li}

Beijing Tiantan Hospital

\section{Wangshu Xu}

Beijing Tiantan Hospital

\section{Xinli Wang}

Beijing Tiantan Hospital

\section{Yunyun Duan}

Beijing Tiantan Hospital

Yaou Liu

Beijing Tiantan Hospital

Xinghu Zhang

Beijing Tiantan Hospital

De-Cai Tian ( $\boldsymbol{\sigma}$ decaitian@hotmail.com )

Beijing Tiantan Hospital https://orcid.org/0000-0002-5153-2491

\section{Research}

Keywords: Neuroimmune, NMOSD, acute attack, MRI, Clinical trial

Posted Date: July 14th, 2020

DOI: https://doi.org/10.21203/rs.3.rs-41052/v1

License: (c) (1) This work is licensed under a Creative Commons Attribution 4.0 International License. Read Full License

Version of Record: A version of this preprint was published at Neurotherapeutics on January 19th, 2021. See the published version at https://doi.org/10.1007/s13311-020-00973-9. 


\section{Abstract}

Background: Gadolinium (Gd)-contrast MRI, reliable detecting blood-brain barrier (BBB) breakdown, is widely used in neuromyelitis optica spectrum disorder (NMOSD) attack. To investigate the predictive value of persistently Gadolinium-enhanced lesions to long-term outcome after attack.

Methods: Data for this analysis come from an ongoing prospective cohort study to assess the clinical and imaging patterns of neuroinflammation diseases in China (CLUE). NMOSD patients with acute attack were enrolled from January 2019 to March 2020. All patients underwent Gadolinium-contrast Magnetic Resonance Imaging (MRI) at baseline and 1 month, and disability was assessed using the Expanded Disability Status Scale (EDSS) at baseline and month 1 and 6. Primary outcome was EDSS score improvement from baseline to month 6 . Multiple logistic regression identified predictors for poor recovery of NMOSD attack.

Results: 41 participants were analyzed, of which 21 patients with persistently Gd-enhancing lesions. Patients in non-continuous enhancement (nCE) group showed statistically significant shift in the 6-month EDSS distributions compared with continuous enhancement (CE) group $(p=0.002)$. Poor recovery rate of CE group was higher than that of nCE group at 6 months $(p=0.033)$. In patients with AQP4-IgG positive, first attack, transverse myelitis or high-dose steroid treatment, the improvement of EDSS scores in CE group was still less compared to nCE group $(p<0.05)$. The presence of persistently Gd-enhancing lesion appears to be associated with poor recovery after acute attack $(\mathrm{OR}=5.473,95 \% \mathrm{Cl} 1.417-21.115, \mathrm{p}=$ $0.014)$.

Conclusion: NMOSD patients with persistently gadolinium-enhancing lesions have worse outcome at 6 months. Persistently Gd-enhancing lesion is a poor prognosis predictor after NMOSD attack.

Trial registration: This clinical trial named Clinical and Imaging Patterns of Neuroinflammation Diseases in China (CLUE), which has registered with ClinicalTrials.gov (NCT04106830). The date of registration was September 27, 2019 (Retrospectively registered). The URL is https://clinicaltrials.gov/ct2/show/NCT04106830?term=NCT04106830\&cntry=CN\&draw=2\&rank=1.

\section{Background}

Neuromyelitis optica spectrum disorder (NMOSD) is humoral immune-mediated astrocytic disease characterized by severe attacks of transverse myelitis and optic neuritis. ${ }^{1}$ Aquaporin-4 antibody (AQP4ab) as a specific marker of NMOSD has paved the way to reveal its pathogenesis. ${ }^{2}$ Acute attacks cause severe disability and require aggressive treatment in patients with NMOSD. ${ }^{3}$ Currently widely recognized first-line treatments mainly includes high dose intravenous corticosteroids ${ }^{4}$ and plasma exchange or adsorption removal AQP-4 antibodies. ${ }^{5}$ However, a certain proportion of patients still present with poor outcomes of functional recovery even with adequate treatment. 
In addition to known prognostic factors of NMOSD attack, such as old-age, high serum IL-6 level, high GFAP level, ${ }^{6-8}$ the breakdown of the blood-brain barrier (BBB) may also a noteworthy factor. The BBB is formed by brain microvascular endothelial cells (BMECs) in conjunction with pericytes and astrocytes, which is important for maintaining brain homeostasis and preventing the entry of AQP4 autoantibody. ${ }^{9}$ Circulating AQP4-ab need to pass through the disrupted BBB in order to reach AQP4 on the astrocyte endfeet to cause the disease. It is therefore important to evaluate the BBB function damage degree in patients with NMOSD attack.

Several methods have been developed to determinate the leakage of the BBB. As the BBB limits the albumin cross from blood to the cerebrospinal fluid (CSF) and albumin is not produced in central nervous system (CNS), the CSF/serum albumin ratio can be used to measure disruption of the BBB. The disruption of the BBB is also characterized by the degradation of the junctional complex proteins and increase in multiple matrix metalloproteinases. ${ }^{10} \mathrm{MRI}$ is a reliable method of detecting BBB breakdown in NMOSD and gadolinium enhancement of lesions on T1-weighted MRI is interpreted as a hallmark of BBB disruption. ${ }^{11}$ In order to understand the predict value of post-contrast MRI continuous enhancement and provide monitoring indicators for subacute sequential treatment, we explored the clinical characteristics and long-term prognosis after acute attack in NMOSD patients with different MRI Gd-enhancement duration.

\section{Methods}

\section{Patients}

We conducted an ongoing prospective study to assess the clinical and imaging patterns of neuroinflammation diseases in China (CLUE). All patients were enrolled between January 2019 to March 2020. NMOSD diagnose was according to 2015 Revised International Criteria. ${ }^{12}$ The inclusion criteria for this study: 1) aged between 15 and 75;2) present with an acute attack; 3) present with gadolinium enhancing lesions in brain, spinal cord or orbit MRI. Acute attack was defined as a new onset of neurological symptoms which were caused by CNS damage and lasted longer than 24 hours. The exclusion criteria for the study:1) initial EDSS score $\geq 6$ before this attack; 2 ) treatment delay more than 3 weeks from symptom onset; 3 ) patients with heart, liver, lung and renal failure and severe infections (e.g. HIV) and malignant tumors; 4) patient is pregnant or nursing; 5) patient had other inflammatory diseases of CNS. The NMOSD patients were assigned into two groups (CE vs. nCE group) according to presence of gadolinium enhancing lesions at 1 month.

All patients were treated with high-dose intravenous methylprednisolone (IVMP) at a dose of $500 \mathrm{mg}$ daily for five consecutive days starting on the day of diagnosis, then reduced to $240 \mathrm{mg}$ for 3 days, followed by $120 \mathrm{mg}$ for 3 days, and finally tapered orally. If clinician determined that high dose IVMP treatment was ineffective (symptoms not improve), more intensive treatment was taken. Intravenous Immunoglobulin (IVIg) was given of $0.4 \mathrm{~g} / \mathrm{kg} /$ day for 5 days as further therapy. Immunoadsorption (IA) 
was performed using tryptophan linked polyvinyl alcohol immusorba TR-350 (Diamed Medizintechnik $\mathrm{GmbH}$, Cologne, Germany) and treated one plasma volume with a maximum of $3000 \mathrm{~mL}$ plasma per session for all patients, adsorption every other day for total of 5 times. Physicians choose different immunosuppressive therapies based on the patient's condition. Prednisone $60 \mathrm{mg} / \mathrm{d}$ was the starting dose, reduce the dose by $5 \mathrm{mg}$ per week. Mycophenolate mofetil (MMF) started $250 \mathrm{mg}$ twice daily, then increased to $500 \mathrm{mg}$ twice daily after 1 week. A course of rituximab (RTX) treatment consists of $500 \mathrm{mg}$ intravenously, repeated 2 weeks and 6 months later.

Written informed consents were provided by all participants or their guardians. This project was approved by the Ethics committee of Beijing Tiantan Hospital, Capital Medical University, Beijing, China. The study has registered with ClinicalTrials.gov (NCT04106830).

\section{Clinical assessment}

Baseline characteristics and clinical features were obtained for all patients recruited, including age of onset, gender, disease duration, treatment profile, etc. Physical disability was determined by the EDSS score at baseline, month 1 and 6 . All EDSS score of the patients was assessed by trained neurologists. We calculated the proportion of EDSS score improvement: (acute - late)/ (acute - prior at attack), which ensure comparability of interscore. Outcome was defined as 'good recovery', 'medium' and 'poor recovery' represent $66 \%-100 \%, 33 \%-66 \%$ and $0-33 \%$ improvement, ${ }^{13}$ respectively. The primary assessment was to examine the effect of gadolinium-enhancing lesions persistently exist on the change in EDSS at followup.

\section{Laboratory analysis}

Serum and CSF samples were collected before treatment. All samples were taken and tested at the biochemical laboratory of Beijing Tiantan Hospital. The permeability of the BBB was determined from the CSF and blood albumin level ratio (Qalb = CSF albumin/serum albumin) ${ }^{14}$ Serum AQP4-IgG and MOGIgG was test by cell-based assay (Oumeng Biotechnology Corporation, Guangzhou, China).

\section{MRI protocol and image analysis}

The patients performed first post-contrast MRI scans of brain, spinal or orbit within 24 hours from the neurologic evaluation and before treatment initiation, and performed second gadolinium enhanced MRI scans at 4 week \pm 3 days. All MRI scans were done on the same 3T Signa scanner (Philips Ingenia CX, Best, The Netherlands). The following MRI sequences were collected: 1) T1-weighted echo( TR = $7 \mathrm{msec}$, $\mathrm{TE}=3 \mathrm{msec}$, slice thickness $=1 \mathrm{~mm}$, matrix $=250 \times 250)$; and 2) T2-weighted echo $(\mathrm{TR}=6816 \mathrm{msec}, \mathrm{TE}=$ $93 \mathrm{msec}$, slice thickness $=6 \mathrm{~mm}$, matrix $=512 \times 512)$; and 3) postcontrast ( 5 minutes after the injection of $0.1 \mathrm{mmol} / \mathrm{Kg} \mathrm{Gd}) \mathrm{T} 1$-weighted echo $(\mathrm{TR}=7 \mathrm{msec}, \mathrm{TE}=3 \mathrm{msec}$, slice thickness $=1 \mathrm{~mm}$, matrix $=250 \times$ 250). An enhancing lesion is defined as an area with hyperintensity on T1-weighted images obtained at least 5 minutes after contrast agent administration, which identified by consensus by two neuroradiologists who were not aware of clinical data (X.W. and Y.D.).

\section{Statistical analyses}


Statistical analysis was conducted by SPSS 25.0 (International Business Machines Corporation, Chicago, IL, USA). Continuous data were reported as mean \pm standard deviation or as median with inter-quartile range (IQR). Categorical variables were presented as absolute and relative frequencies.

We analyzed the differences between two groups (CE vs nCE group) using the Chi-square test or Fisher's exact test for categorical variables. Student's t-tests and Mann-Whitney $\mathrm{U}$ tests were adopted as parametric and nonparametric tests for continuous variables. Ordinal Logistic Regression was adopted to analyzed the distribution of EDSS and improvement graded of two groups. Wilcoxon signed-rank test was used to analysis of the EDSS score improve. Multivariate logistic regression analyses were used to identify independent factors for distinguishing between two groups. The two-tailed $p$ value $<0.05$ was considered statistically significant.

\section{Data availability}

According to consent provided by the participants, any unpublished and anonymized data will be shared from corresponding author on request from qualified investigators.

\section{Results}

\section{Patients}

We screened 138 individuals for enrollment to the study, of whom 73 were judged ineligible or declined to participate. Of the 65 patients who enrolled into the study, 47 patients completed twice MRI scans as required, after the exclusion of 18 patients who completed MRI scan not meet the criteria or had not undergone MRI assessment after 1 month. Patients were assigned into continuous enhancement and non-continuous enhancement groups depending on their MRI images. Of the 44 patients who completed follow-up of 6 months, 41 were included in the final analysis after the exclusion of 3 patients who had relapsed within 6 months. All 3 patients with relapsed had increased EDSS scores ( 1.5 to 3.5 points), and the lesions were located in the cervical spinal cord or optic nerve (Fig. 1).

\section{Clinical and demographic features}

A total of 41 NMOSD patients were analyzed, of whom 21 (51.2\%) patients were assigned into Continuous Enhancement group and 20 (48.8\%) patients were non-Continuous Enhancement group. The sex ratio, median age, disease duration, ARR were similar between two groups. A total of $25(61 \%)$ patients were first attack, 9 (22\%) were second attack, the remainder had more than 3 times relapses. Median delay prior to acute attack treatment was 14 days in CE group, similar with that in nCE group ( $p=$ 0.168). There were 16 (76.2\%) AQP4-IgG seropositive patients in CE group, while 17 (85.0\%) in nCE group. Thirty-three NMOSD attacks were treated with IVMP alone, 4 attacks were treated with IVMP + IVIg and 4 attacks were treated with IVMP + Immunoadsorption (Table 1). 
Table 1

Demographic and clinical characteristics of NMOSD patients according to MRI group.

\begin{tabular}{|c|c|c|c|}
\hline & CE group $(n=21)$ & nCE group $(n=20)$ & $\mathbf{P}$ \\
\hline Age, mean (SD), years & $41.4(16.4)$ & $40.6(16.5)$ & 0.874 \\
\hline Female, n (\%) & $15(71.4)$ & $17(85.0)$ & 0.454 \\
\hline Disease duration, median (IQR), months & $2(0.8-12.0)$ & $1.5(1-8.2)$ & 0.927 \\
\hline Annual relapse rate, mean (SD) & $1.4(1.3)$ & $1.7(1.4)$ & 0.602 \\
\hline First attack, n (\%) & $13(62.0)$ & $12(60.0)$ & 0.901 \\
\hline Second attack, n (\%) & $4(19.0)$ & $5(25.0)$ & 0.719 \\
\hline$\geq 3$ attacks, n (\%) & $4(19.0)$ & $3(15.0)$ & 0.731 \\
\hline Delay from onset to treatment, median (IQR), days & $14(12-20.5)$ & $13.5(8.5-16)$ & 0.168 \\
\hline AQP4-IgG positive, n (\%) & $16(76.2)$ & $17(85.0)$ & 0.697 \\
\hline MOG-IgG positive, n (\%) & $2(9.5)$ & $0(0)$ & 0.488 \\
\hline Qalb $\left(\times 10^{-3}\right)$, median (IQR) & $6.5(4.7-13.1)$ & $5.4(3.9-7.0)$ & 0.069 \\
\hline Clinical manifestation, n (\%) & & & 0.717 \\
\hline Optic neuritis & $1(4.8)$ & $2(10.0)$ & \\
\hline LETM & $17(80.9)$ & $13(65.0)$ & \\
\hline Brain & $1(4.8)$ & $1(5.0)$ & \\
\hline Brainstem & $2(9.5)$ & $4(20.0)$ & \\
\hline Coexisting autoimmune Diseases, n (\%) & $8(38.1)$ & $3(15.0)$ & 0.093 \\
\hline Treatment, n (\%) & & & 0.844 \\
\hline IVMP & $16(76.2)$ & $17(85.0)$ & \\
\hline IVMP + IVlg & $2(9.5)$ & $2(10.0)$ & \\
\hline IVMP + IA & $3(14.3)$ & $1(5.0)$ & \\
\hline Relapse prevention treatment, $\mathrm{n}(\%)$ & & & 0.328 \\
\hline oral prednisone + RTX & $8(38.1)$ & $6(30.0)$ & \\
\hline oral prednisone + MMF & $1(4.8)$ & $2(10.0)$ & \\
\hline oral prednisone & $12(57.1)$ & $9(45.0)$ & \\
\hline Not use & $0(0)$ & $3(15.0)$ & \\
\hline
\end{tabular}




\section{CE group $(n=21) \quad n C E$ group $(n=20) \quad P$}

Abbreviations: NMOSD = neuromyelitis optica spectrum disorder; CE = continuous enhancement; $\mathrm{nCE}$ = non-continuous enhancement; SD = standard deviation; IQR = inter-quartile range; $\mathrm{AQP} 4$ = Aquaporin 4; MOG = myelin oligodendrocyte glycoprotein; Qalb = CSF albumin/serum albumin; LETM = Longitudinal Extensive Transverse Myelitis; IVMP = Intravenous methylprednisolone therapy; IVIg = Intravenous immunoglobulin; IA = Immunoadsorption; RTX = Rituximab; MMF = Mycophenolate Mofetil.

\section{MRI characteristics}

A total of 82 scans for 41 patients, of which 65 gadolinium-enhancing lesions were included in the analysis. Spinal cord lesions involved were as follows: 22 in cervical, 9 in thoracic, 14 in cervical-thoracic, and 2 lumbosacral of lesions. Six brain lesions mainly involving frontal Lobe, corpus callosum, basal ganglia, and cerebral peduncle. $47(72.3 \%)$ of the gadolinium-enhancing lesions affecting the spinal cord while 4 (6.2\%) located in optic nerve, 8 (12.3\%) in brainstem and $6(9.2 \%)$ in brain. 20 lesions (48.8\%) were characterized by a disappearance of gadolinium-enhancing voxels on post-contrast T1-weighted imaging. By contrast, there were 21 (51.2\%) persistent enhancing lesions. Typical MRI images of two groups exhibit in Fig. 2.

\section{Disability recovery during 6-month follow-up}

The EDSS ranges from 0 to 10 (divided into 6 groups), with higher scores indicating increased disability. We noted EDSS score improved in two groups, but distribution shifts showed significant difference between two groups after one-month $(P=0.011)$. Furthermore, patients in nCE group showed a significant shift in the 6-month EDSS distributions compared with CE group (OR = 8.890, 95\% $\mathrm{Cl} 2.227-35.516, \mathrm{p}=$ 0.002) (Fig. 3).

The proportion of 'good recovery' were similar between two groups at one month $(p=0.120)$. After sixmonths of follow-up, only $14.3 \%$ of CE group gained 'good recovery' while $50 \%$ of nCE group reached 'good recovery'. Poor recovery rate of CE group was higher than that of nCE group (OR $=3.654,95 \% \mathrm{Cl}$ 1.111-12.001, $p=0.033$ ) (Fig. 3).

\section{EDSS change in patients with AQP4-IgG positive, first attack, LETM and IVMP treatment}

In thirty-three patients with AQP4-IgG positive, median EDSS decreased from 4.5 to 3 in CE group at 6 months while nCE group decrease from 4 to 2. Median EDSS of nCE group was lower than CE group after one-month $(p=0.02)$ and the gap widened between two groups at six months $(p=0.001)$ (Fig. 4).

25 patients experienced first attack with median EDSS decreased from 4.0 to 3.5 at one-month and 2.5 at 6 months in CE group. By contrast, the median EDSS score for patients in nCE group dropped from 3.5 to 2 at 6 months. In patients with first episode, the improvement of EDSS in nCE group after one-month follow-up was significant compared to CE group $(p=0.032)$, and the trend continued to 6 months $(p=$ 0.042) (Fig. 4). 
We observed disability improved in 30 patients with Longitudinal Extensive Transverse Myelitis (LETM) during follow-up. Median EDSS decreased from 4 to 3.5 at one-month and further to 3 at six-months in CE group. Correspondingly, disability measured by EDSS improved from 3 to 2 points after one-month followup and remained at 2 at 6 months in nCE group. Compare to CE group, lower median EDSS of nCE group was observed after one-month $(p=0.034)$ and the trend continued to six months $(p=0.001)$ (Fig. 4$)$.

In 33 patients treated only with IVMP, median EDSS decreased from 4 to 3 in CE group compared with 3.5 to 2 in nCE group at 6 months. Median EDSS of nCE group was lower than CE group after one-month ( $p=$ 0.027 ) and the gap between two groups was even more pronounced at six months ( $p=0.003)$ (Fig. 4).

\section{Predict factors of long-term recovery}

Multivariate logistic regression analysis was used to assess the predict factors of the recovery of NMOSD patients. The result of analysis using the following 4 factors: persistently gadolinium enhancing lesions, AQP4-IgG positive, first attack and Qalb. Only two factors were significantly: persistently enhancing lesions $(\mathrm{OR}=5.473,95 \% \mathrm{Cl} 1.417-21.115, \mathrm{p}=0.014)$, first attack $(\mathrm{OR}=0.226,95 \% \mathrm{Cl} 0.059-0.862, \mathrm{p}=$ 0.029) (Table 2).

Table 2

Multivariate logistic regression analysis of prognosis predicts factors in NMOSD attack

\begin{tabular}{|lllc|}
\hline Factor & OR & $95 \% \mathrm{Cl}$ & P value \\
\hline Persistently enhancing lesions & 5.473 & $(1.417-21.115)$ & $0.014^{*}$ \\
\hline AQP4-IgG positive & 0.823 & $(0.162-4.178)$ & 0.814 \\
\hline First attack & 0.226 & $(0.059-0.862)$ & $0.029^{*}$ \\
\hline Qalb & 1.073 & $(0.956-1.203)$ & 0.225 \\
\hline $\begin{array}{l}\text { Abbreviations: NMOSD = neuromyelitis optica spectrum disorder; Qalb = CSF albumin/serum albumin; } \\
\text { OR = odds ratio; Cl = confidence interval. * }{ }^{\circ}<0.05 \text { represent statistical significance. }\end{array}$ \\
\hline
\end{tabular}

\section{Discussion}

This study investigated the impact of gadolinium-enhancing lesions persistently exist on the prognosis of NMOSD attack. We found that physical disability improvement in CE group was less than that of nCE group at 1 month, and the trend continued to 6 months. In patients with AQP4-IgG positive, first attack, LETM or IVMP treatment, median EDSS of nCE group was lower than CE group after 6 months follow-up. We have confirmed that persistent existence gadolinium enhancing lesions were predictors of poor prognosis in NMOSD attack.

All NMOSD patients in our cohort were experiencing acute attack with enhanced lesions. The presence of gadolinium-enhancing lesions indicate that contrast agent has entered the CNS via compromised BBB, considering gadolinium compounds do not readily cross a stable BBB. ${ }^{15}$ Hence enhancing lesions of 
demyelinating patients reflects the destruction of the BBB. ${ }^{16,17}$ Several signs on activity and persistence of lesions have been obtained in vivo in NMOSD patients by contrast MRI. In our series of NMOSD patients, enhancement was displayed in poorly marginated and multiple patchy patterns mainly in the lateral ventricle, optic nerve or the cervical and upper thoracic medulla. The enhanced lesions described above, so called "cloud-like" enhancement of brain lesions, chiasmal enhancement of optic neuritis, and ring enhancement of the spinal cord are more common in NMOSD patients. ${ }^{18,19}$

We observed that the distribution of EDSS score shifted not significant in CE group than nCE group at one-month and six-months. Meanwhile, we also found the "good recovery" proportion of CE group was lower than that of nCE group. Disruption of BBB may be the pathological mechanism for poor prognosis of patients in CE group. The BBB is a crucial barrier that limits brain entry of peripheral cells and potentially harmful molecules. The persistent existence of gadolinium-enhancing lesions indicates that BBB is still open, and peripheral AQP4 antibodies and inflammatory factors may still enter the lesions leading to damage. ${ }^{20}$ Inflammatory mediators can disrupt the BBB then AQP4 antibodies or other inflammatory factors entry into CNS thus initiate pathological process of NMOSD. ${ }^{21}$ As disability improved, only $55.43 \%$ of new enhancing lesions gradually disappear within 3 weeks. ${ }^{22}$ The increased BBB permeability is an important feature of NMOSD patients, then it is crucial to assess disruption of BBB. The level of matrix metalloproteinase -2/9 (MMP-2/9) were confirmed as biomarkers of BBB disruption. ${ }^{23,24}$ However, lumbar puncture is invasive and patients are less susceptible to dynamic continuous screening. Dynamic contrast enhanced-MRI (DCE-MRI) provides semi-quantitative and quantitative data to accurately assess the permeability of $\mathrm{BBB},{ }^{25}$ but it has disadvantage of long scanning time (15 minutes) and requires unique post progress methods. By contrast, enhanced MRI is a reliable and convenient method of detecting BBB breakdown.

In addition, several factors were taken into account that may impact the prognosis of NMOSD patients. High dose steroids is the mainstay therapy for acute relapses that target widely cellular part of inflammation, and PELX or IA appears very promising in eliminating specific antibodies, complement and proinflammatory factors. ${ }^{26,27}$ The benefit of early initiation of PLEX was confirmed rather than as a rescue therapy only after steroid failure. ${ }^{13}$ However, only 3 patients in CE group and 1 patient in nCE group treated by IA, the subgroup analysis was performed only for all IVMP treatment. EDSS improved poorly in CE group compared to nCE group in patients treated with IVMP. Longitudinal extensive transverse myelitis presented with markedly higher EDSS scores than brain-dominant type in patients with NMOSD. ${ }^{28}$ LETM characterized by symptoms and signs of neurologic dysfunction in motor and sensory tracts on both sides of spinal cord. Using EDSS to measure disability in LETM is more accurate, since it is sensitive to assess physical disabilities. ${ }^{29}$ Therefore, we conducted LETM subgroup analysis, while 3 patients with optic neuritis, 8 patients with brain/brainstem phenotypes did not analyze separately. In LETM subgroup analysis, CE group experienced poorer improvement in disability compared with nCE group. Patients with first-attack tend to have favorable outcome and our analysis of patients with first-attack also found better prognosis in the nCE group. 
We found that persistently gadolinium enhancing lesion is important factors in predicting the prognosis of NMOSD attack. It is important to closely follow-up of NMOSD patients with acute attacks and adjust or escalate treatment timely. ${ }^{30,31}$ Furthermore, most patients with NMOSD attack have only $19.1 \%$ complete remission rates after first course of treatment, which may associated with lack of adequate short-term follow-up after treatment of an attack ${ }^{5}$. There are several MRI imaging features, including T1 hypointensity, cavitation or atrophy, indicates that patients with NMOSD are more likely to have a high risk of poor recovery and permanent disability. ${ }^{18,32}$ Usually, most of these predictors reflect chronic changes of disease, and cannot provide more information for therapy escalation effectively. Thus, it becomes important to assessed the BBB function in acute phase by contrast MRI.

Our study has several limitations. First, present study was limited by small sample size, and future highquality researches with bigger sample size are required to confirm the conclusions of the current study. We did not evaluate NFL, GFAP, or cytokines in serum or CSF since this was beyond scope of our current study. Furthermore, it should be noted that our current study does not provide quantitative assessment of BBB permeability, but we have gradually carried out DCE-MRI studies that can more accurately assess BBB leakage.

\section{Conclusion}

Our data suggest that persistently enhancing lesions might be useful in determining long-term poor recovery after NMOSD attack and that more intensive treatment or immunosuppressive therapy might be warranted if the patient has persistently gadolinium enhancing lesions.

\section{Abbreviations}

\section{AQP4-ab}

Aquaporin-4 antibody

BBB

blood-brain barrier

\section{BMECs}

brain microvascular endothelial cells

CE

continuous enhancement

Cl

confidence interval

CNS

central nervous system

CSF

cerebrospinal fluid

DCE-MRI

Dynamic contrast enhanced-MRI 


\section{EDSS}

Expanded Disability Status Scale

Gd

Gadolinium

IA

Immunoadsorption

IVlg

intravenous Immunoglobulin

IVMP

intravenous methylprednisolone

IQR

inter-quartile range

LETM

Longitudinal Extensive Transverse Myelitis

MMF

Mycophenolate mofetil

MMP-2/9

matrix metalloproteinase $-2 / 9$

MOG

myelin oligodendrocyte glycoprotein

MRI

Magnetic Resonance Imaging

NMOSD

neuromyelitis optica spectrum disorder

nCE

non-continuous enhancement

OR

odds ratio

Qalb

CSF albumin/serum albumin

RTX

rituximab

SD

standard deviation

TR

repetition time

TE

time of echo

Declarations 
Ethics approval and consent to participate: This project was approved by the Ethics committee of Beijing Tiantan Hospital, Capital Medical University, Beijing, China. (KY 2019-050-02)

Consent for publication: Not applicable.

Availability of data and materials: The datasets used or analyzed during the current study are available from the corresponding author on reasonable request.

Competing interests: none declared.

Funding: Work in the authors' laboratories was supported in part by grants from National Natural Science Foundation of China (81801199).

Authors' contributions: D.C.T and X.Z formulated the conception and design of this study; X.L, and W.X contributed to acquisition and analysis of clinical data; X.W. and Y.D. contributed to acquisition and analysis of MRI data; Y.L. revised the manuscript; Y.X drafted the manuscript and prepared the figures.

Acknowledgements: We thank our patients for participating in this study, and all the members of Jing-Jin Neuroimmunology Teams for recruiting patients and other support.

\section{References}

1. Weinshenker BG, Wingerchuk DM. Neuromyelitis Spectrum Disorders. Mayo Clin Proc. 2017;92:66379. DOI:10.1016/j.mayocp.2016.12.014.

2. Lennon VA, Wingerchuk DM, Kryzer TJ, et al. A serum autoantibody marker of neuromyelitis optica: distinction from multiple sclerosis. The Lancet. 2004;364:2106-12. DOI:10.1016/s01406736(04)17551-x.

3. Collongues N, Ayme-Dietrich E, Monassier L, et al. Pharmacotherapy for Neuromyelitis Optica Spectrum Disorders: Current Management and Future Options. Drugs. 2019;79:125-42.

DOI:10.1007/s40265-018-1039-7.

4. Elsone L, Panicker J, Mutch $\mathrm{K}$, et al. Role of intravenous immunoglobulin in the treatment of acute relapses of neuromyelitis optica: experience in 10 patients. Mult Scler. 2014;20:501-4.

DOI:10.1177/1352458513495938.

5. Kleiter I, Gahlen A, Borisow N, et al. Neuromyelitis optica: Evaluation of 871 attacks and 1,153 treatment courses. Ann Neurol. 2016;79:206-16. DOI:10.1002/ana.24554.

6. Seok JM, Cho HJ, Ahn SW, et al. Clinical characteristics of late-onset neuromyelitis optica spectrum disorder: A multicenter retrospective study in Korea. Mult Scler. 2017;23:1748-56. DOI:10.1177/1352458516685416.

7. Takano R, Misu T, Takahashi T, et al. Astrocytic damage is far more severe than demyelination in NMO: a clinical CSF biomarker study. Neurology. 2010;75:208-16.

DOI:10.1212/WNL.0b013e3181e2414b. 
8. Bergamaschi R, Ghezzi A. Devic's neuromyelitis optica: clinical features and prognostic factors. Neurol Sci. 2004;25(Suppl 4):364-7. DOl:10.1007/s10072-004-0342-0.

9. Shimizu F, Nishihara H, Kanda T. Blood-brain barrier dysfunction in immuno-mediated neurological diseases. Immunol Med. 2018;41:120-8. DOI:10.1080/25785826.2018.1531190.

10. Uchida T, Mori M, Uzawa A, et al. Increased cerebrospinal fluid metalloproteinase-2 and interleukin- 6 are associated with albumin quotient in neuromyelitis optica: Their possible role on blood-brain barrier disruption. Mult Scler. 2017;23:1072-84. DOI:10.1177/1352458516672015.

11. Waubant E. Biomarkers indicative of blood-brain barrier disruption in multiple sclerosis. Dis Markers. 2006;22:235-44. DOI:10.1155/2006/709869.

12. Wingerchuk DM, Banwell B, Bennett JL, et al. International consensus diagnostic criteria for neuromyelitis optica spectrum disorders. Neurology. 2015;85:177-89.

DOI:10.1212/WNL.0000000000001729.

13. Bonnan M, Valentino R, Debeugny S, et al. Short delay to initiate plasma exchange is the strongest predictor of outcome in severe attacks of NMO spectrum disorders. J Neurol Neurosurg Psychiatry. 2018;89:346-51. DOI:10.1136/jnnp-2017-316286.

14. Freedman MS, Thompson EJ, Deisenhammer F, et al. Recommended standard of cerebrospinal fluid analysis in the diagnosis of multiple sclerosis: a consensus statement. Arch Neurol. 2005;62:86570. DOI:10.1001/archneur.62.6.865.

15. Albrecht DS, Granziera C, Hooker JM, et al. In Vivo Imaging of Human Neuroinflammation. ACS Chem Neurosci. 2016;7:470-83. DOI:10.1021/acschemneuro.6b00056.

16. Grossman RI, Braffman BH, Brorson JR, et al. Multiple sclerosis: serial study of gadolinium-enhanced MR imaging. Radiology. 1988;169:117-22. DOI:10.1148/radiology.169.1.3420246.

17. Katz D, Taubenberger JK, Cannella B, et al. Correlation between magnetic resonance imaging findings and lesion development in chronic, active multiple sclerosis. Ann Neurol. 1993;34:661-9. DOI:10.1002/ana.410340507.

18. Kim HJ, Paul F, Lana-Peixoto MA, et al. MRI characteristics of neuromyelitis optica spectrum disorder: an international update. Neurology. 2015;84:1165-73. DOI:10.1212/WNL.0000000000001367.

19. Yokote $\mathrm{H}$, Nose $\mathrm{Y}$, Ishibashi S, et al. Spinal cord ring enhancement in patients with neuromyelitis optica. Acta Neurol Scand. 2015;132:37-41. DOI:10.1111/ane.12354.

20. Obermeier B, Daneman R, Ransohoff RM. Development, maintenance and disruption of the bloodbrain barrier. Nat Med. 2013;19:1584-96. DOI:10.1038/nm.3407.

21. Guo Y, Weigand SD, Popescu BF, et al. Pathogenic implications of cerebrospinal fluid barrier pathology in neuromyelitis optica. Acta Neuropathol. 2017;133:597-612. DOI:10.1007/s00401-0171682-1.

22. Cotton $F$, Weiner HL, Jolesz FA, et al. MRI contrast uptake in new lesions in relapsing-remitting MS followed at weekly intervals. Neurology. 2003;60:640-6. DOI:10.1212/01.wnl.0000046587.83503.1e. 
23. Tomizawa Y, Yokoyama $\mathrm{K}$, Saiki S, et al. Blood-brain barrier disruption is more severe in neuromyelitis optica than in multiple sclerosis and correlates with clinical disability. J Int Med Res. 2012;40:148391. DOI:10.1177/147323001204000427.

24. Hosokawa T, Nakajima H, Doi Y, et al. Increased serum matrix metalloproteinase-9 in neuromyelitis optica: implication of disruption of blood-brain barrier. J Neuroimmunol. 2011;236:81-6. DOI:10.1016/j.jneuroim.2011.04.009.

25. Cramer SP, Modvig S, Simonsen HJ, et al. Permeability of the blood-brain barrier predicts conversion from optic neuritis to multiple sclerosis. Brain. 2015;138:2571-83. DOl:10.1093/brain/awv203.

26. Abboud H, Petrak A, Mealy M, et al. Treatment of acute relapses in neuromyelitis optica: Steroids alone versus steroids plus plasma exchange. Mult Scler. 2016;22:185-92. DOI:10.1177/1352458515581438.

27. Lipphardt M, Muhlhausen J, Kitze B, et al. Immunoadsorption or plasma exchange in steroidrefractory multiple sclerosis and neuromyelitis optica. J Clin Apher. 2019;34:381-91. DOI:10.1002/jca.21686.

28. Nagaishi A, Takagi M, Umemura A, et al. Clinical features of neuromyelitis optica in a large Japanese cohort: comparison between phenotypes. J Neurol Neurosurg Psychiatry. 2011;82:1360-4. DOI:10.1136/jnnp-2011-300403.

29. Meyer-Moock S, Feng YS, Maeurer M, et al. Systematic literature review and validity evaluation of the Expanded Disability Status Scale (EDSS) and the Multiple Sclerosis Functional Composite (MSFC) in patients with multiple sclerosis. BMC Neurol. 2014;14:58. DOI:10.1186/1471-2377-14-58.

30. Kimbrough DJ, Fujihara K, Jacob A, et al. Treatment of Neuromyelitis Optica: Review and Recommendations. Mult Scler Relat Disord. 2012;1:180-7. DOI:10.1016/j.msard.2012.06.002.

31. Sellner J, Boggild M, Clanet M, et al. EFNS guidelines on diagnosis and management of neuromyelitis optica. Eur J Neurol. 2010;17:1019-32. DOI:10.1111/j.1468-1331.2010.03066.x.

32. Cassinotto $\mathrm{C}$, Deramond $\mathrm{H}$, Olindo $\mathrm{S}$, et al. MRI of the spinal cord in neuromyelitis optica and recurrent longitudinal extensive myelitis. J Neuroradiol. 2009;36:199-205.

DOI:10.1016/j.neurad.2008.12.008.

\section{Figures}




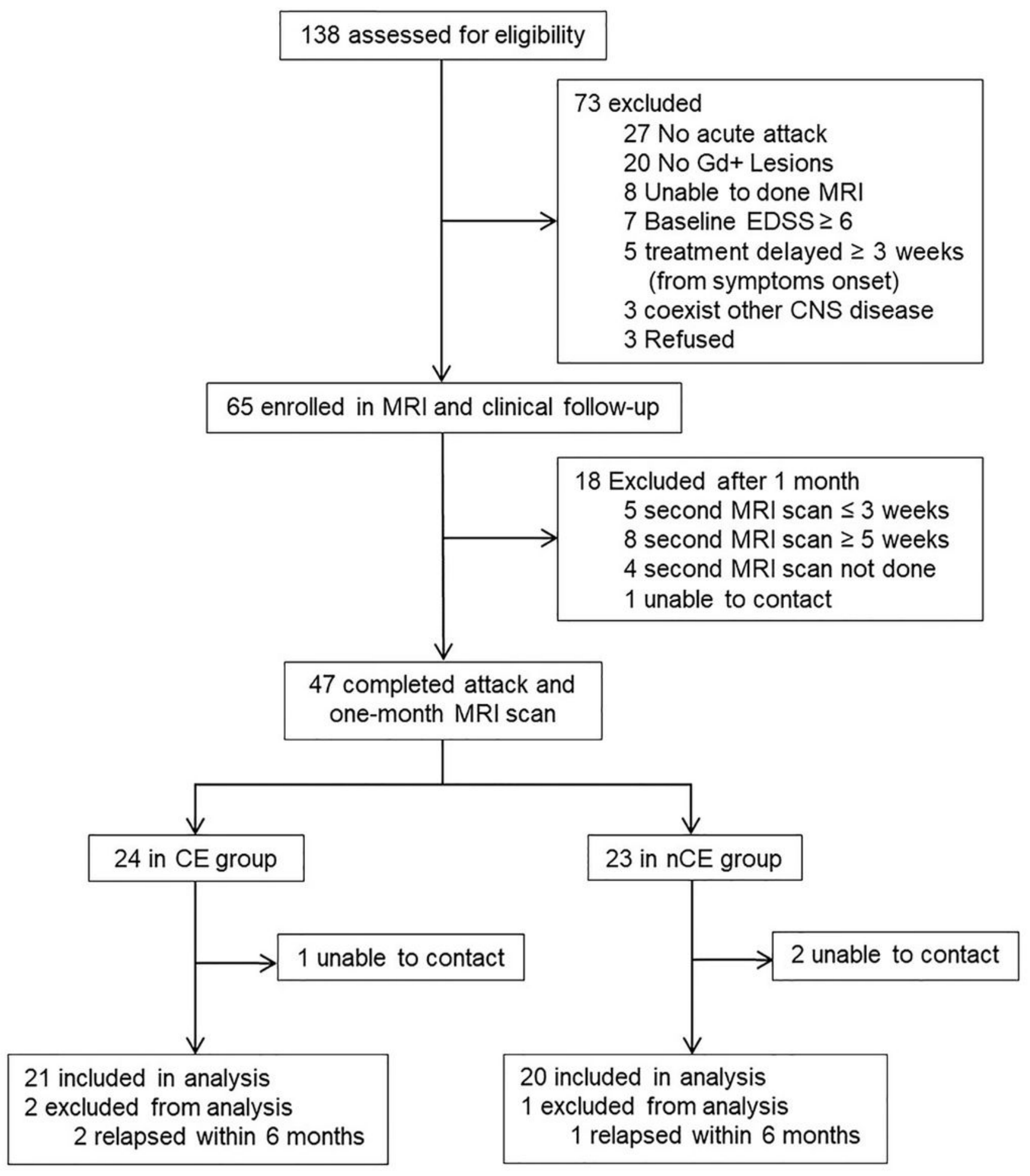

Figure 1

Trial profile of the post-contrast MRI scan trial. Abbreviations: Gd+ = gadolinium-positive; EDSS = Expanded Disability Status Scale; CNS = central never system; CE = continuous enhancement; $\mathrm{nCE}$ = noncontinuous enhancement. 


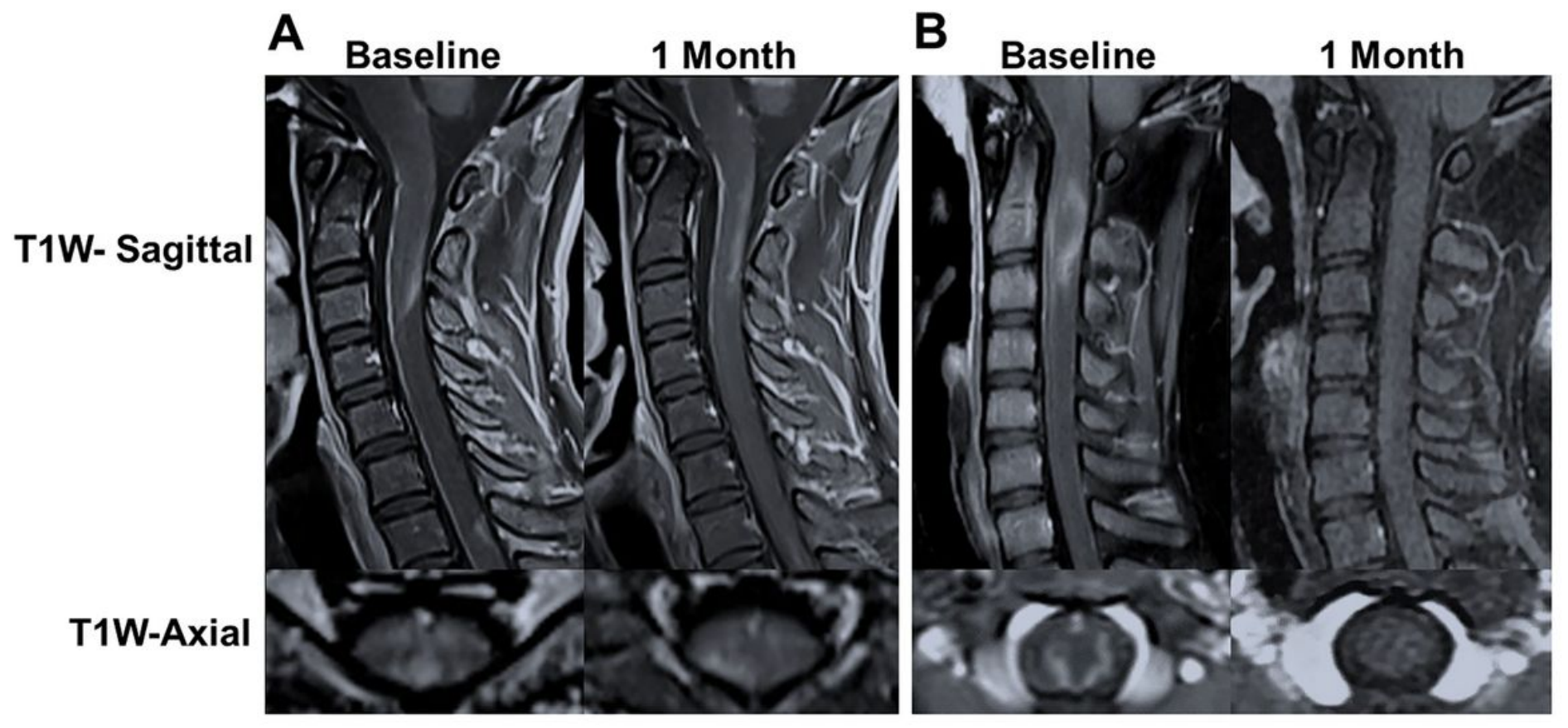

Figure 2

Representative gadolinium enhancement MRI images of NMOSD patients. (A) Patchy gadoliniumenhanced lesion was found in $\mathrm{C} 1-3$ and persisted exist after one-month. (B) Open-ring enhanced lesion in C1-3 disappeared at one-month follow up.

A

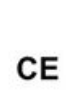

CE

$$
\begin{aligned}
& \text { Attack } \\
& 1 \text { Month }
\end{aligned}
$$$$
23.8 \%
$$

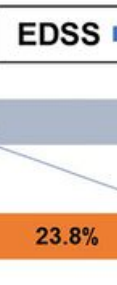

6 Month

$4.8 \%$
$33.3 \%$

nCE

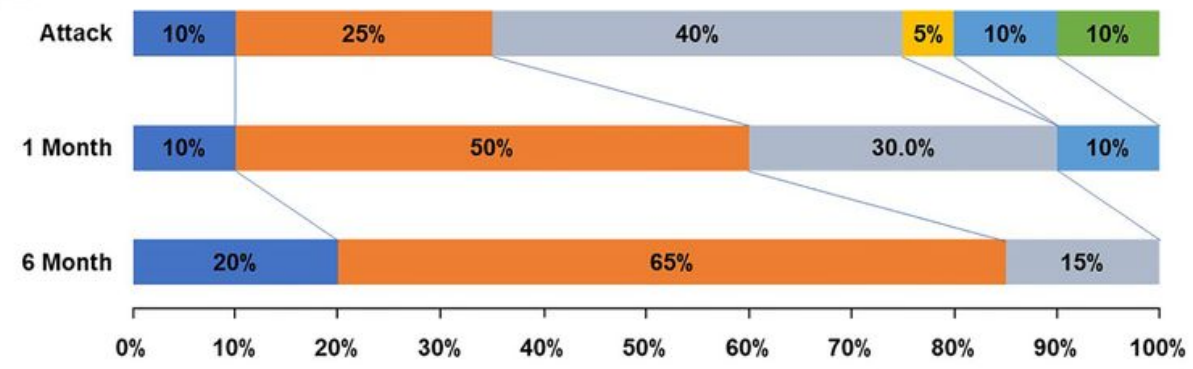

B

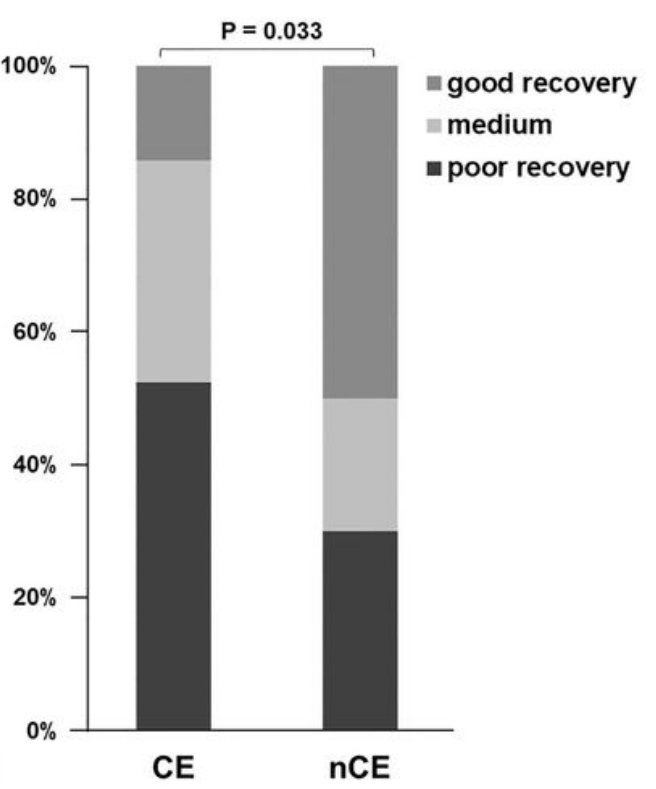

Figure 3

The changes of EDSS score in CE and nCE group after follow up. (A) Distribution of Expanded Disability Status Scale (EDSS) scores at attack, at 1 month and at 6 months in two groups. (B) The improvement of 
EDSS score in the continuous enhancement group was worse than that in the non-continuous enhancement group at 6 months.
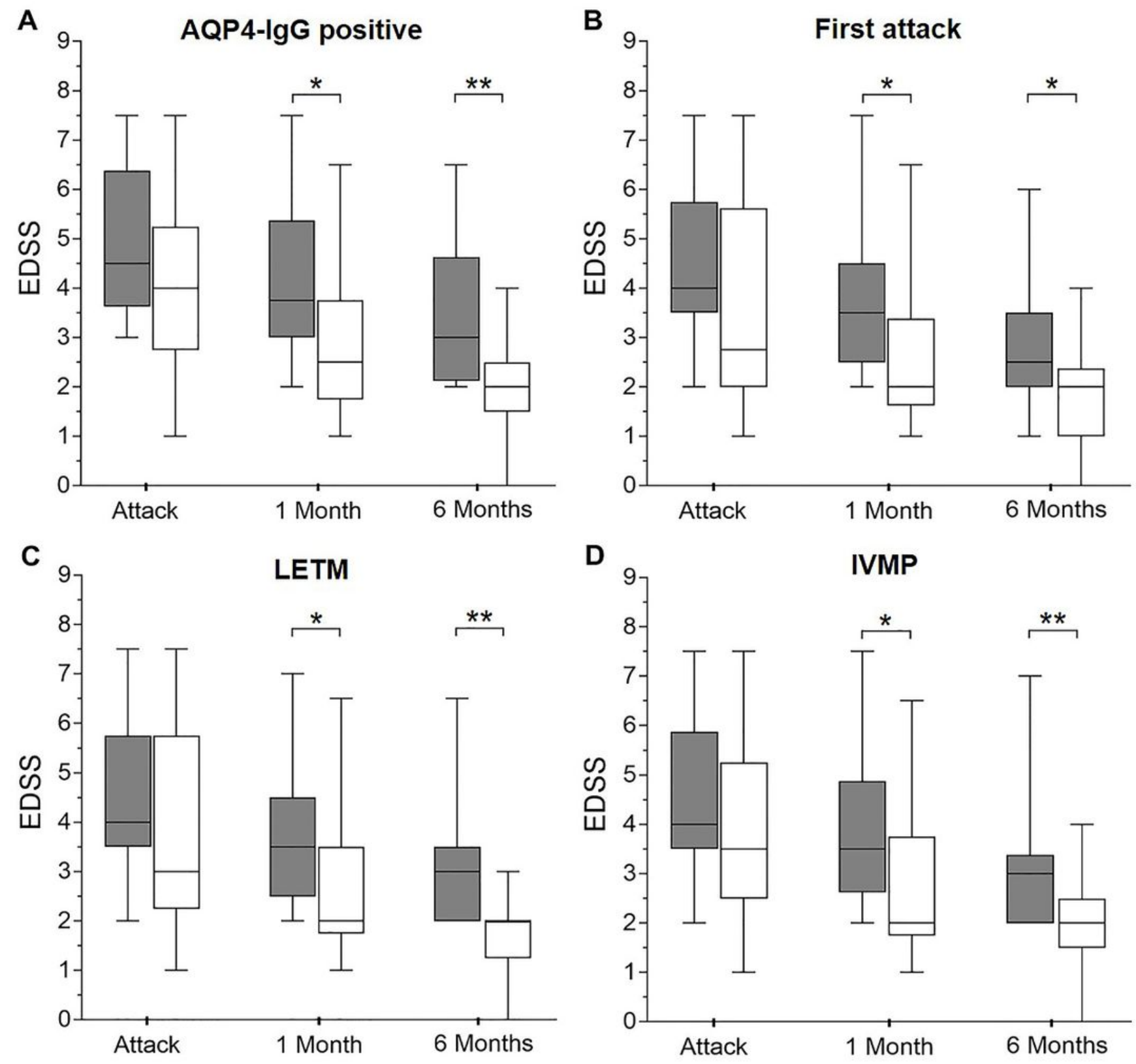

\section{Figure 4}

Comparison of EDSS score changes in patients with AQP4-IgG positive, first attack, LETM, IVMP treatment (A) Median EDSS score of nCE group was lower in patients with AQP4-IgG positive at six-month $(n=33)$. (B) EDSS improvement in CE group was less than nCE group in Patients who experienced first attack ( $n=25)$. (C) In patients with transverse myelitis, EDSS improved poorly in CE group compared to $\mathrm{nCE}$ group $(\mathrm{n}=30)$. (D) In patients who treated only with IVMP凹six-month Median EDSS score was lower in $\mathrm{nCE}$ group $(\mathrm{n}=33) . * \mathrm{p}<0.05$ and $* * \mathrm{p}<0.01$ represent statistical significance. 